\title{
LoC-SERS Combined with the Standard Addition Method: Toward the Quantification of Nitroxoline in Spiked Human Urine Samples
}

Izabella J. Hidi, ${ }^{1,2} \ddagger$ Martin Jahn, ${ }^{1,2}+$ Karina Weber, ${ }^{1,2,4}$ Thomas Bocklitz, ${ }^{1}$ Mathias W. Pletz, ${ }^{3,4}$ Dana Cialla-May, ${ }^{1,2,4^{*}}$ Juergen Popp $^{1,2,4}$

${ }^{1}$ Friedrich Schiller University Jena, Institute of Physical Chemistry and Abbe Center of Photonics, Helmholtzweg 4, 07745 Jena, Germany

${ }^{2}$ Leibniz Institute of Photonic Technology Jena, Albert-Einstein-Str. 9, 07745 Jena, Germany

${ }^{3}$ Center for Infectious Diseases and Infection Control, Jena University Hospital, Erlanger Allee 10107740 Jena

${ }^{4}$ Research Campus Infectognostic, Philosophenweg 7, 07743 Jena, Germany, Germany

*corresponding author: Phone: +49 (0)3641-206309; Fax: +49 (0)3641-206399, e-mail: dana.cialla-may@uni-jena.de

\section{Supporting Information}

Table of Content:

Table S1 Sample preparation - Urinary NTX concentrations measured for sample HV1, HV2 and HV3;

Table S2 Sample preparation - LoC-SERS-SAM;

Table S3 Band assignment of the observed Raman modes of NTX;

Figure S1: UV-Vis measurements;

Figure S2: Normalized Raman and SERS spectrum of NTX powder and $100 \mu$ M solution;

Figure S3: LoC-SERS spectra measured with conventional configuration.

Figure S4: The chemical affinity of NTX toward Ag NPs: SERS spectra of various mixtures;

Figure S5: Reference SERS spectrum of NTX vs. LoC-SERS signal of the blank urine and of NTX spiked in the urine sample HV1;

Figure S6: Mean SERS spectra and their double standard deviation of urine samples from healthy volunteer and UTI patients;

Table S4, Table S-5 and Table S-6: LoC-SERS-SAM results;

Figure S7: MCR-ALS plots,

Table S7: correlation coefficients for MCR-ALS. 
Table S1. Sample preparation - Urinary NTX concentrations measured for sample HV1, HV2 and HV3.

\begin{tabular}{|c|c|c|}
\hline $\mathrm{c}_{\mathrm{NTX} / \mathrm{H} 2 \mathrm{O}}[\mu \mathrm{M}]$ & $\mathrm{c}_{\mathrm{NTX} / \text { in-droplet }}[\mu \mathrm{M}]$ & $\mathrm{c}_{\mathrm{NTX} / \text { urine }}[\mu \mathrm{M}]$ \\
\hline 10 & 3.0 & 4.28 \\
\hline 17.5 & 5.25 & 7.50 \\
\hline 25 & 7.50 & 10.70 \\
\hline 40 & 12.00 & 17.10 \\
\hline 55 & 16.50 & 23.50 \\
\hline 70 & 21.00 & 30.00 \\
\hline 85 & 25.50 & 36.40 \\
\hline 100 & 30.00 & 42.80 \\
\hline
\end{tabular}

Obs.: NTX/H $\mathrm{H}_{2} \mathrm{O}$ solution at different concentrations ( $\left.c_{N T X / H 2 O}\right)$ was mixed with the urine sample in 3:7 ratio. $c_{N T X / i n-d r o p l e t}$ represents the concentration of NTX in the total volume $\left(V_{N T X / H 2 O}+V_{\text {urine }}\right)$, while $c_{N T X / u r i n e}$ is the NTX concentration in the volume of urine.

Table S2. Sample preparation - LoC-SERS-SAM

\begin{tabular}{|c|c|c|c|c|}
\hline Sample & $\mathrm{V}_{\text {urine }}[\mu \mathrm{l}]$ & $V_{\text {spike }}[\mu l]$ & $\mathbf{V}_{\text {standard }}[\boldsymbol{\mu l}]$ & $\mathrm{V}_{\mathrm{H} 2 \mathrm{O}}[\mu \mathrm{I}]$ \\
\hline blank & \multirow{6}{*}{250} & 0 & 0 & 250 \\
\hline unknown & & \multirow{5}{*}{76} & 0 & 174 \\
\hline SAS1 & & & 43.4 & 130.6 \\
\hline SAS2 & & & 87 & 87 \\
\hline SAS3 & & & 130.6 & 43.4 \\
\hline SAS4 & & & 174 & 0 \\
\hline
\end{tabular}

for $\mathrm{c}_{\text {spike }}=69.4 \mu \mathrm{M}$ and $\mathrm{c}_{\text {standard }}=57.4 \mu \mathrm{M}(\mathrm{HV} 4$ and HV5)

\begin{tabular}{|c|c|c|c|c|c|c|c|}
\hline Sample & $\begin{array}{l}\mathbf{n}_{\text {spike }} \\
{[\mathrm{mol}]}\end{array}$ & $\begin{array}{c}\mathbf{n}_{\text {standard }} \\
{[\mathrm{mol}]}\end{array}$ & $\begin{array}{l}\mathbf{n}_{\text {total }} \\
{[\mathbf{m o l}]}\end{array}$ & $\begin{array}{c}\mathbf{c}_{\mathrm{NTX} / \text { urine }} \\
{[\mathbf{M}]}\end{array}$ & $\begin{array}{c}\mathbf{c}_{\mathrm{NTX} / \text { urine }} \\
{[\mathrm{mg} / \mathrm{l}]}\end{array}$ & $\begin{array}{c}\mathbf{c}_{\mathrm{NTX} \text { in-droplet }} \\
{[\mathbf{M}]}\end{array}$ & $\begin{array}{l}\mathbf{c}_{\mathrm{SAS}} \\
{[\mathbf{M}]}\end{array}$ \\
\hline unknown & \multirow{5}{*}{$1.05 \cdot 10^{-8}$} & 0 & $1.05 \cdot 10^{-8}$ & $2.10 \cdot 10^{-5}$ & 4 & $1.05 \cdot 10^{-5}$ & 0 \\
\hline SAS1 & & $4.99 \cdot 10^{-9}$ & $1.55 \cdot 10^{-8}$ & $3.10 \cdot 10^{-5}$ & 5.89 & $1.55 \cdot 10^{-5}$ & $4.99 \cdot 10^{-6}$ \\
\hline SAS2 & & $9.99 \cdot 10^{-9}$ & $2.05 \cdot 10^{-8}$ & $4.10 \cdot 10^{-5}$ & 7.79 & $2.05 \cdot 10^{-5}$ & $9.98 \cdot 10^{-6}$ \\
\hline SAS3 & & $1.50 \cdot 10^{-8}$ & $2.55 \cdot 10^{-8}$ & $5.10 \cdot 10^{-5}$ & 9.69 & $2.55 \cdot 10^{-5}$ & $1.49 \cdot 10^{-5}$ \\
\hline SAS4 & & $2.00 \cdot 10^{-8}$ & $3.05 \cdot 10^{-8}$ & $6.10 \cdot 10^{-5}$ & 11.59 & $3.05 \cdot 10^{-5}$ & $1.99 \cdot 10^{-5}$ \\
\hline
\end{tabular}

for $\mathrm{c}_{\text {spike }}=70 \mu \mathrm{M}$ and $\mathrm{c}_{\text {standard }}=60 \mu \mathrm{M}$ (PS1-PS5 )

\begin{tabular}{|c|c|c|c|c|c|c|c|}
\hline Sample & $\begin{array}{l}\mathbf{n}_{\text {spike }} \\
{[\mathrm{mol}]}\end{array}$ & $\begin{array}{c}\mathbf{n}_{\text {standard }} \\
\text { [mol] }\end{array}$ & $\begin{array}{l}\mathbf{n}_{\text {total }} \\
{[\mathrm{mol}]}\end{array}$ & $\begin{array}{c}\mathbf{c}_{\mathrm{NTX} / \text { urine }} \\
{[\mathrm{M}]}\end{array}$ & $\begin{array}{c}\mathbf{c}_{\mathrm{NTX} / \text { urine }} \\
{[\mathrm{mg} / \mathrm{l}]}\end{array}$ & $\begin{array}{c}\mathbf{c}_{\text {NTX in-droplet }} \\
{[\mathrm{M}]}\end{array}$ & $\begin{array}{l}\mathbf{c}_{\mathrm{SAS}} \\
{[\mathrm{M}]}\end{array}$ \\
\hline unknown & \multirow{5}{*}{$5.32 \cdot 10^{-9}$} & 0 & $5.32 \cdot 10^{-9}$ & $2.13 \cdot 10^{-5}$ & 4.04 & $1.06 \cdot 10^{-5}$ & 0 \\
\hline SAS1 & & $2.60 \cdot 10^{-9}$ & $7.92 \cdot 10^{-9}$ & $3.17 \cdot 10^{-5}$ & 6.02 & $1.58 \cdot 10^{-5}$ & $5.21 \cdot 10^{-6}$ \\
\hline SAS2 & & $5.22 \cdot 10^{-9}$ & $1.05 \cdot 10^{-8}$ & $4.22 \cdot 10^{-5}$ & 8.01 & $2.11 \cdot 10^{-5}$ & $1.04 \cdot 10^{-5}$ \\
\hline SAS3 & & $7.84 \cdot 10^{-9}$ & $1.32 \cdot 10^{-8}$ & $5.26 \cdot 10^{-5}$ & 10.00 & $2.63 \cdot 10^{-5}$ & $1.57 \cdot 10^{-5}$ \\
\hline SAS4 & & $1.04 \cdot 10^{-8}$ & $1.58 \cdot 10^{-8}$ & $6.30 \cdot 10^{-5}$ & 11.98 & $3.15 \cdot 10^{-5}$ & $2.09 \cdot 10^{-5}$ \\
\hline
\end{tabular}


Table S3. Band assignment of the observed Raman modes of NTX. ${ }^{1}$

\begin{tabular}{c|c}
$\begin{array}{c}\text { Position } \\
{\left[\mathbf{c m}^{-1}\right]}\end{array}$ & Assignment \\
\hline 421 & CCC out-of-plane bending \\
\hline 490 & C-O in-plane bending \\
\hline 565 & CCC in-plane bending \\
\hline 729 & O-H out-of-plane bending \\
\hline 800 & aromatic C-H out-of-plane bending \\
\hline 840 & aromatic C-H in-plane bending \\
\hline 987 & C-N stretching \\
\hline 1153 & C-NO ${ }_{2}$ stretching \\
\hline 1197 & C-O stretching \\
\hline 1249 & O-H in-plane bending \\
\hline 1313 & C=N stretching \\
\hline 1405 & C=C stretching \\
\hline 1465 & \\
\hline 1573 & \\
\hline
\end{tabular}

\section{Droplet based microfluidic chip}

The microfluidic platform is fabricated from glass via chemical etching and anodic binding and has a size of 16x25 mm. Glass has a low Raman scattering cross section, therefore it is an ideal material for manufacturing microfluidic chips for SERS measurements. Prior to the measurements, the surface of the micro-channels was functionalized with octadecyltrichlorosilane (ODTS) in order to obtain hydrophobic channel walls. The microfluidic platform has the following operational units: a droplet generator where the aqueous solutions are dispensed into the flow of mineral oil via a T-junction, a dosing unit where further aqueous solutions are injected into the already existing droplet, two meander channels assuring an optimal mixing of the droplet content and a long measurement loop. Generally, through port 1-3 the analyte solutions and the solvent (purified water, water or urine containing the target analyte) are pumped, while the $\mathrm{Ag}$ nanoparticles and $\mathrm{KCl}$, as aggregation agent, are injected via port 4 and 5 . The flow rate of the continuous phase was fixed at $9 \mathrm{nl} / \mathrm{s}$, the sum of the flowrates at the droplet generator was $14 \mathrm{nl} / \mathrm{s}$ and at the dosing unit $11 \mathrm{nl} / \mathrm{s}$. The various solutions are filled into glass syringes that are connected with the chip via Teflon capillaries. The software provided with the pump system (neMESYS Cetoni $\mathrm{GmbH}$ ) controls the flow rates and allows the preparation of droplets containing differently concentrated samples, while maintaining the droplet volume constant.

\section{UV-Vis measurements}

The UV-Vis spectra plotted in Figure S1 show the extinction profile of the bare Ag NPs, of the NTX molecule at $100 \mu \mathrm{M}$ and the extinction bands of the Ag NPs and NTX mixture without and with $\mathrm{KCl}$ addition. The maximum of the extinction band due to the single particle resonance is centered at $408 \mathrm{~nm}$ proving the successful synthesis. ${ }^{2,3}$ The NTX molecule has several absorption bands. The detailed assignment of the bands is behind the purpose of the present study. When Ag NPs are mixed with NTX in a 1 to 1 ratio, the UV-Vis spectrum presents a broad band centered at $423 \mathrm{~nm}$. This is the result of the convolution of the absorption band of the Ag NPs and 
of the two bands of NTX at $363 \mathrm{~nm}$ and $448 \mathrm{~nm}$. In order to achieve high SERS enhancements "hot-spots" are needed. Thus, the single spherical nanoparticles have to be aggregated after the analyte molecule is absorbed on the metallic surface. For this, $1 \mathrm{M} \mathrm{KCl}$ solution was added in the following ratio: Ag NPs:NTX:KCl=1:1:0.1. As a consequence, in the UV-Vis spectrum the single particle resonance is diminishing in intensity and a second broad band appears at higher wavelengths. This is associated with coupled localized surface plasmon resonances originating from two or more closely related nanoparticles. The connection between the extinction spectrum and the magnitude of the electromagnetic SERS enhancements is in general indirect. The highest enhancement is obtained from the small gaps in between nanoparticles, rather from the surface of the single ones.

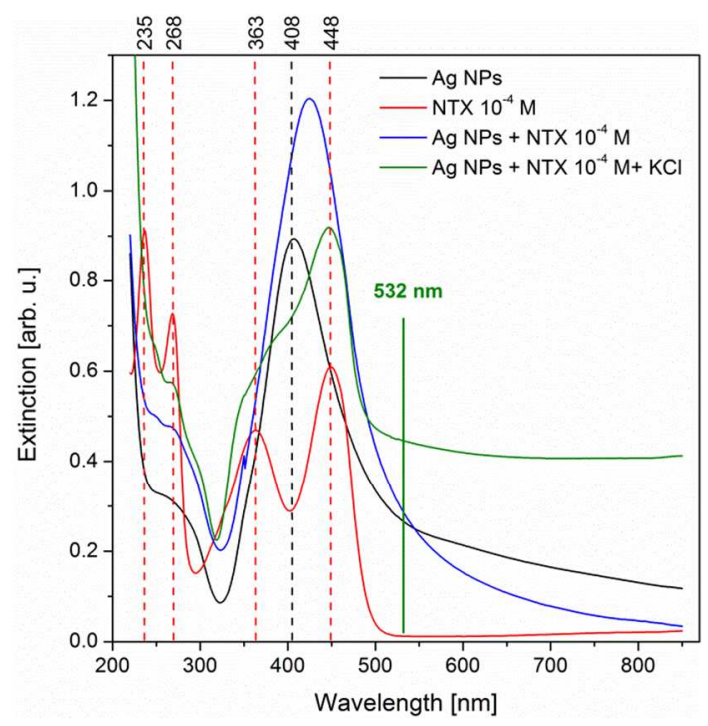

Figure S1. UV-Vis absorption spectra of Ag NPs, of NTX/ $\mathrm{H}_{2} \mathrm{O}$ at $10^{-4} \mathrm{M}$ and of the non-aggregated and aggregated Ag NPs and NTX mixtures. (Ag NPs:NTX:KCl=1:1:0.1)

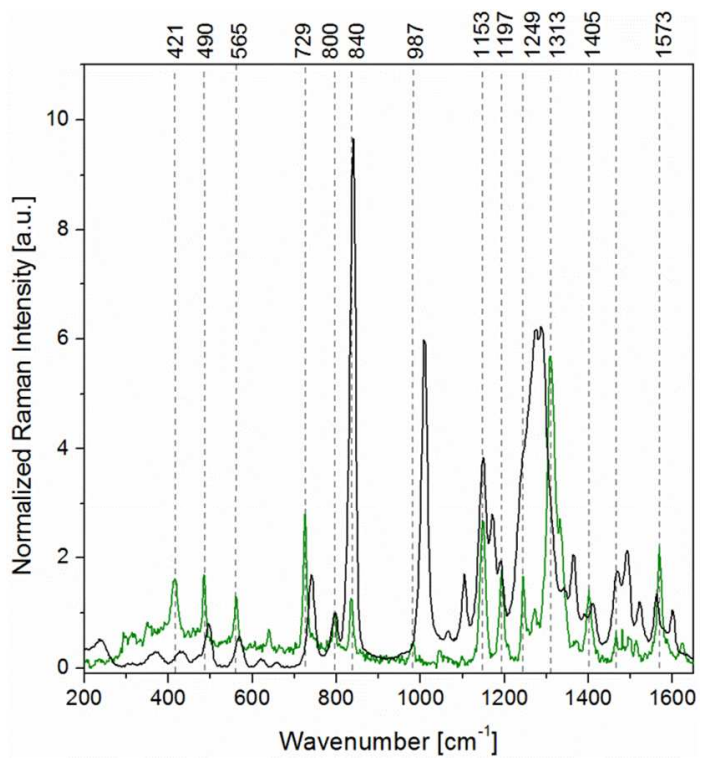

Figure S2. Normalized Raman (green) and SERS (black) spectrum of NTX powder and of an $100 \mu \mathrm{M}$ solution. 


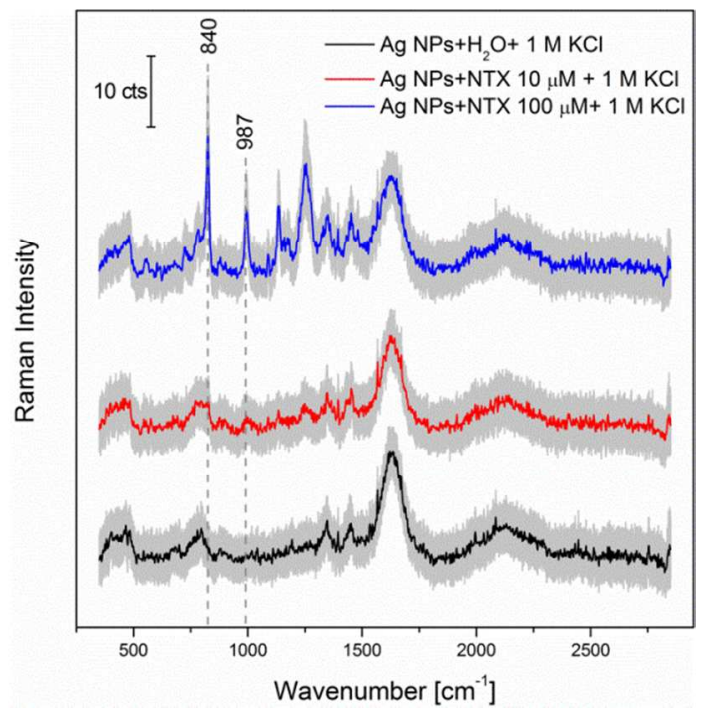

Figure S3. SERS spectra measured with the conventional configuration: port 1: $\mathrm{NTX} / \mathrm{H}_{2} \mathrm{O}$, port 3: purified water, port 4: Ag NPs and port 5: $1 \mathrm{M} \mathrm{KCl}$ (chip design in Scheme 2).

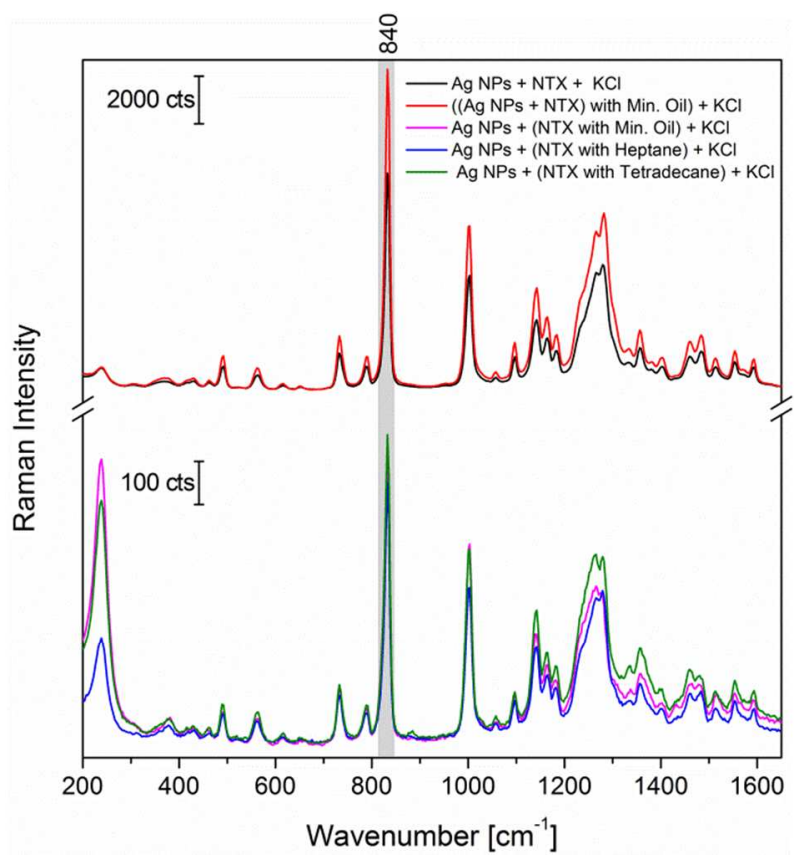

Figure S4. The chemical affinity of NTX toward Ag NPs: SERS spectra of various mixtures. NTX concentration was $100 \mu \mathrm{M}$. 


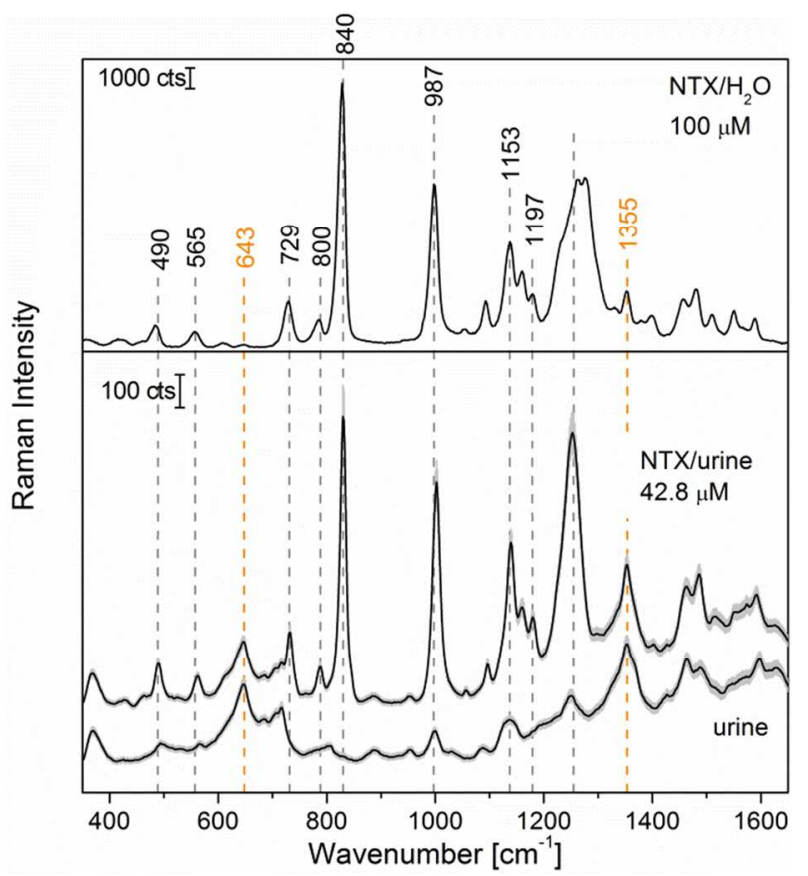

Figure S5. Reference SERS spectrum of NTX vs. LoC-SERS signal of the blank urine and of NTX spiked in the urine sample HV1. 


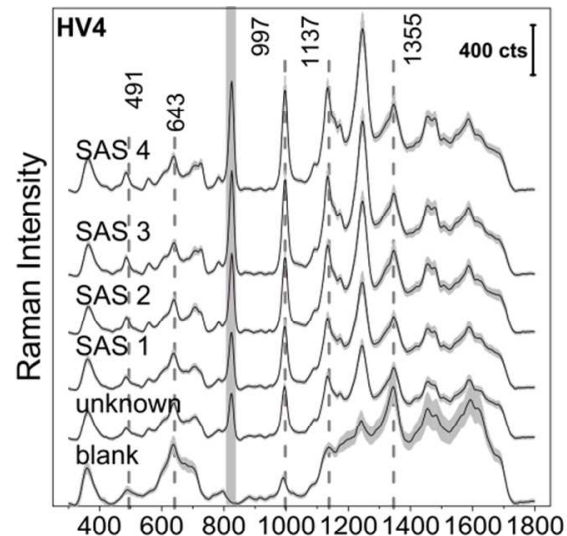

Wavenumber $\left[\mathrm{cm}^{-1}\right]$

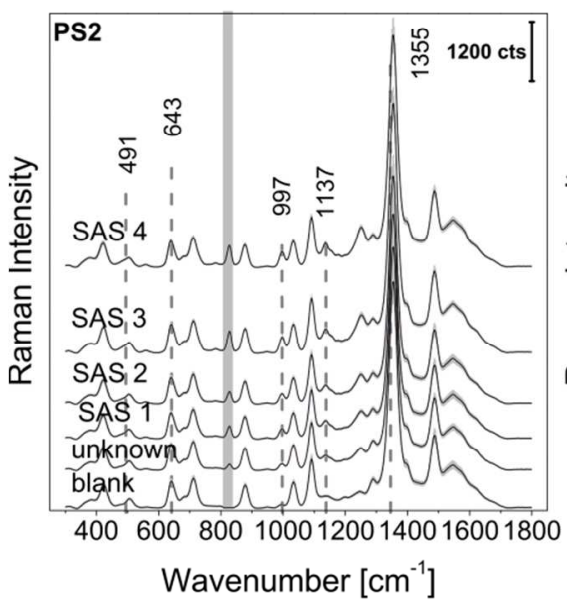

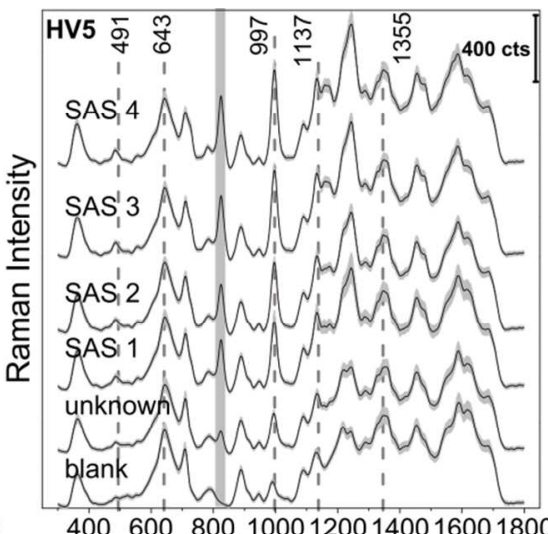

Wavenumber $\left[\mathrm{cm}^{-1}\right]$
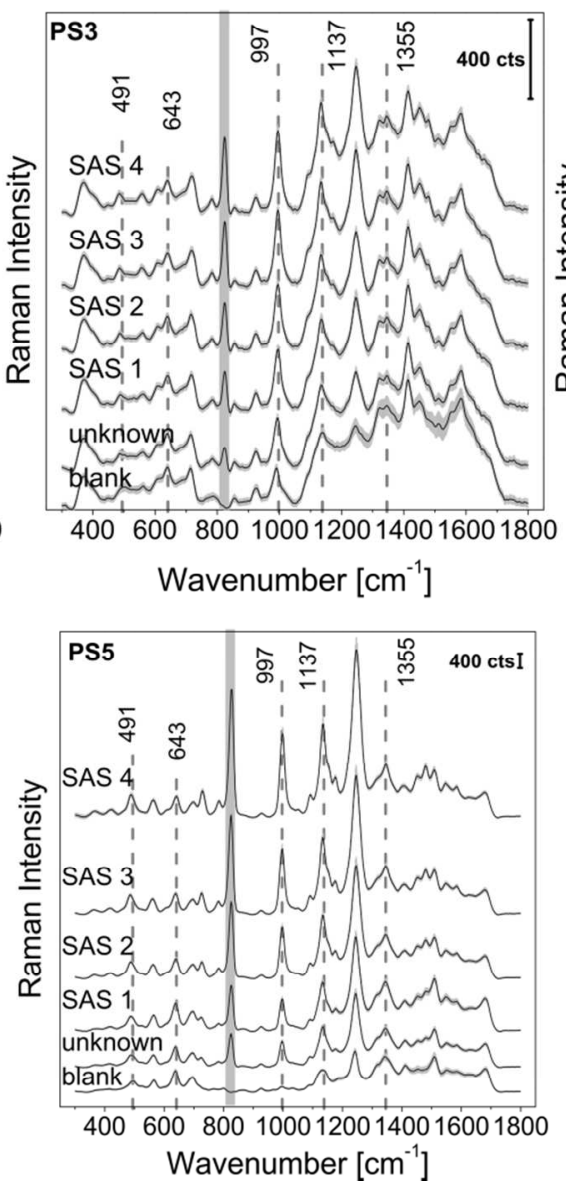

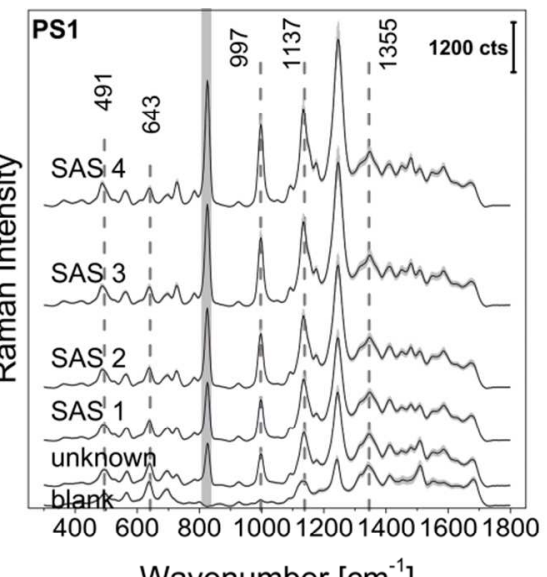

Wavenumber $\left[\mathrm{cm}^{-1}\right]$

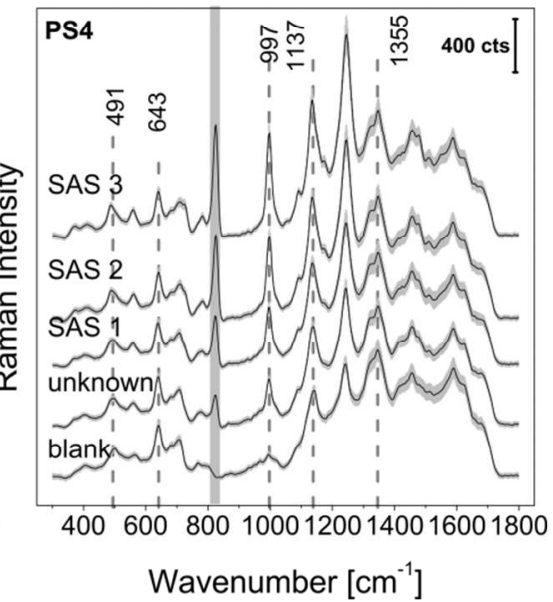

Figure S6. Mean SERS spectra and their double standard deviation of urine samples from healthy volunteer (HV) and UTI patients (PS). 
Table S4. LoC-SERS-SAM results for the Raman band at $\sim \mathbf{8 4 0} \mathrm{cm}^{-1}$ based on univariate statistical analysis and their corresponding $95 \%$ confidence intervals.

\begin{tabular}{|c|c|c|c|c|c|}
\hline Sample & $\mathrm{c}_{0}$ & $\mathrm{E}_{\mathrm{r}}$ & \multicolumn{2}{|c|}{$\mathrm{c}_{\text {unknown }}$} & \multirow{2}{*}{$\begin{array}{c}\mathrm{c}_{\mathrm{NC}} \\
{[\mu \mathrm{M}]}\end{array}$} \\
\cline { 4 - 5 } & {$[\%]$} & {$[\mu \mathrm{M}]$} & {$[\mathrm{mg} / \mathrm{l}]$} & \\
\hline HV4 & $13.2 \pm 1.0$ & 25.6 & $26.4 \pm 2.1$ & $5.0 \pm 0.4$ & $2.1 \pm 0.5$ \\
\hline HV5 & $12.1 \pm 1.1$ & 15.0 & $24.1 \pm 2.2$ & $4.6 \pm 0.4$ & $2.7 \pm 0.6$ \\
\hline PS1 & $9.9 \pm 0.6$ & 6.1 & $19.7 \pm 1.3$ & $3.7 \pm 0.2$ & $0.1 \pm 0.3$ \\
\hline PS2 & $9.1 \pm 0.8$ & 14.2 & $18.2 \pm 1.7$ & $3.5 \pm 0.3$ & $0.5 \pm 0.5$ \\
\hline PS3 & $9.0 \pm 0.5$ & 14.7 & $18.1 \pm 1.1$ & $3.4 \pm 0.2$ & $0.7 \pm 0.4$ \\
\hline PS4 & $7.5 \pm 0.4$ & 29.2 & $15.0 \pm 0.9$ & $2.9 \pm 0.2$ & $1.1 \pm 0.2$ \\
\hline PS5 & $5.8 \pm 0.4$ & 45.1 & $11.6 \pm 0.7$ & $2.2 \pm 0.1$ & $1.6 \pm 0.2$ \\
\hline
\end{tabular}

$\mathrm{c}_{0}$-in-droplet NTX concertation; $\mathrm{c}_{\text {unknown }}$-urinary NTX concertation; $\mathrm{c}_{\mathrm{NC}}$ : predicted NTX concentration for the blank sample.

Table S5. LoC-SERS-SAM results for the Raman band at $\sim 1000 \mathrm{~cm}^{-1}$ based on univariate statistical analysis and their corresponding $95 \%$ confidence intervals.

\begin{tabular}{|c|c|c|c|c|c|}
\hline Sample & $\mathrm{c}_{0}$ & \multirow{2}{*}{$\begin{array}{c}\mathrm{E}_{\mathrm{r}} \\
{[\mu \mathrm{M}]}\end{array}$} & \multicolumn{2}{|c|}{$\mathrm{c}_{\text {unknown }}$} & \multirow{2}{*}{$\begin{array}{c}\mathrm{c}_{\mathrm{NC}} \\
{[\mu]}\end{array}$} \\
\cline { 4 - 5 } & & {$[\mu \mathrm{M}]$} & {$[\mathrm{mg} / \mathrm{l}]$} & \\
\hline HV4 & $25.5 \pm 1.6$ & 142.7 & $51.0 \pm 3.1$ & $9.7 \pm 0.6$ & $18.6 \pm 1.1$ \\
\hline HV5 & $20.7 \pm 1.4$ & 97.5 & $41.5 \pm 2.7$ & $7.9 \pm 0.5$ & $14.1 \pm 0.9$ \\
\hline PS1 & $14.5 \pm 0.8$ & 37.9 & $29.0 \pm 1.5$ & $5.5 \pm 0.3$ & $4.9 \pm 0.4$ \\
\hline PS2 & $26.9 \pm 1.9$ & 153.7 & $53.8 \pm 3.7$ & $10.2 \pm 0.7$ & $20.7 \pm 1.3$ \\
\hline PS3 & $58.0 \pm 2.9$ & 447.6 & $116.1 \pm 5.9$ & $22.1 \pm 1.1$ & $48.3 \pm 1.8$ \\
\hline PS4 & $23.1 \pm 1.1$ & 117.6 & $46.1 \pm 2.1$ & $8.8 \pm 0.4$ & $19.1 \pm 0.8$ \\
\hline PS5 & $9.1 \pm 0.4$ & 14.0 & $18.2 \pm 0.9$ & $3.5 \pm 0.2$ & $2.3 \pm 0.4$ \\
\hline
\end{tabular}

$\mathrm{c}_{0}$-in-droplet NTX concertation; $\mathrm{c}_{\text {unknown }}$-urinary NTX concertation; $\mathrm{c}_{\mathrm{NC}}$ : predicted NTX concentration for the blank sample. 
Table S6. LoC-SERS-SAM results based on MCR-ALS analysis and their corresponding $95 \%$ confidence intervals (Student's t-test).

\begin{tabular}{|c|c|c|c|c|c|}
\hline Sample & $\mathrm{c}_{0}$ & $\mathrm{E}_{\mathrm{r}}$ & \multicolumn{2}{|c|}{$\mathrm{c}_{\text {unknown }}$} & \multirow{2}{*}{$\begin{array}{c}\mathrm{c}_{\mathrm{NC}} \\
{[\mu \mathrm{M}]}\end{array}$} \\
\cline { 4 - 5 } & {$[\mu]$} & {$[\mu \mathrm{M}]$} & {$[\mathrm{mg} / \mathrm{l}]$} & \\
\hline HV4 & $14.0 \pm 1.0$ & 33.9 & $27.9 \pm 2.1$ & $5.3 \pm 0.4$ & $1.8 \pm 0.5$ \\
\hline HV5 & $10.1 \pm 0.9$ & 3.8 & $20.2 \pm 1.7$ & $3.8 \pm 0.3$ & $0.5 \pm 0.5$ \\
\hline PS1 & $10.1 \pm 0.6$ & 4.0 & $20.2 \pm 1.2$ & $3.8 \pm 0.2$ & $1.1 \pm 0.3$ \\
\hline PS2 & $9.8 \pm 1.0$ & 7.8 & $19.5 \pm 2.1$ & $3.7 \pm 0.4$ & $1.9 \pm 0.3$ \\
\hline PS3 & $11.5 \pm 0.6$ & 8.9 & $23.1 \pm 1.1$ & $4.4 \pm 0.2$ & $0.0 \pm 0.3$ \\
\hline PS4 & $5.9 \pm 0.4$ & 43.7 & $11.9 \pm 0.8$ & $2.3 \pm 0.2$ & $0.6 \pm 0.6$ \\
\hline PS5 & $5.6 \pm 0.4$ & 46.9 & $11.3 \pm 0.8$ & $2.1 \pm 0.2$ & $0.8 \pm 0.3$ \\
\hline
\end{tabular}

$\mathrm{c}_{0}$-in-droplet NTX concertation; $\mathrm{c}_{\text {unknown }}$-urinary NTX concertation; $\mathrm{c}_{\mathrm{NC}}$ : predicted NTX concentration for the blank sample. 

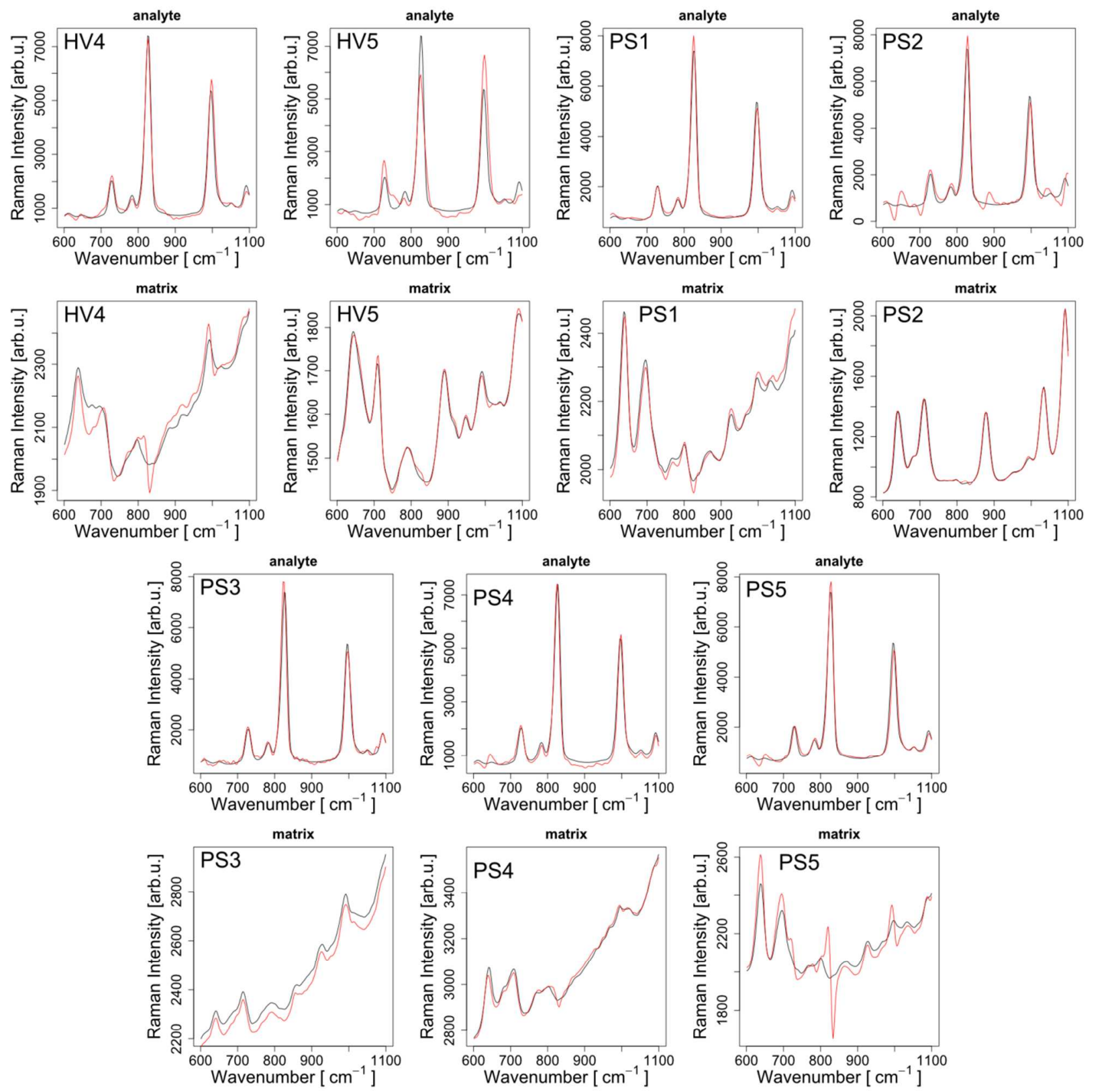

Figure S7. Reference (black) and MCR-ALS recovered (red) spectra of NTX in water (analyte) and pure urine (matrix). 
Table S7. Pearson correlation coefficient of between reference and MCR-ALS recovered spectra.

\begin{tabular}{|c|c|c|}
\hline Sample & $\mathrm{r}_{\text {matrix }}$ & $\mathrm{r}_{\text {analyte }}$ \\
\hline HV4 & 0.965 & 0.988 \\
\hline HV5 & 0.997 & 0.940 \\
\hline PS1 & 0.989 & 0.991 \\
\hline PS2 & 0.997 & 0.969 \\
\hline PS3 & 0.999 & 0.979 \\
\hline PS4 & 0.997 & 0.989 \\
\hline PS5 & 0.899 & 0.987 \\
\hline
\end{tabular}

References:

[1] Arjunan, V.; Balamourougane, P. S.; Kalaivani, M.; Raj, A.; Mohan, S. Spectrochimica Acta Part A: Molecular and Biomolecular Spectroscopy 2012, 96, 506-516.

[2] Leopold, N.; Lendl, B. The Journal of Physical Chemistry B 2003, 107, 5723-5727.

[3] Zhang, C.; Man, B. Y.; Jiang, S. Z.; Yang, C.; Liu, M.; Chen, C. S.; Xu, S. C.; Qiu, H. W.; Li, Z. Appl. Surf. Sci. 2015, 347, 668-672 